\title{
Vom Operationsstress zum Vibrationsbad - der klinische Alltag schreibt Medizingeschichte
}

\author{
Tanya Karrera, \\ Pascal Zehnder ${ }^{b}$ \\ a lic. phil., Sammlungsverant- \\ wortliche Institut für \\ Medizingeschichte Bern \\ und Museale Sammlung der \\ Inselspital-Stiftung
}

b PD Dr. med., Oberarzt I, Universitätsklinik für Urologie, Inselspital Bern
Korrespondenz: Tanya Karrer

Institut für Medizingeschichte Bühlstrasse 26 CH-3012 Bern

tanya.karrer[at]img.unibe.ch

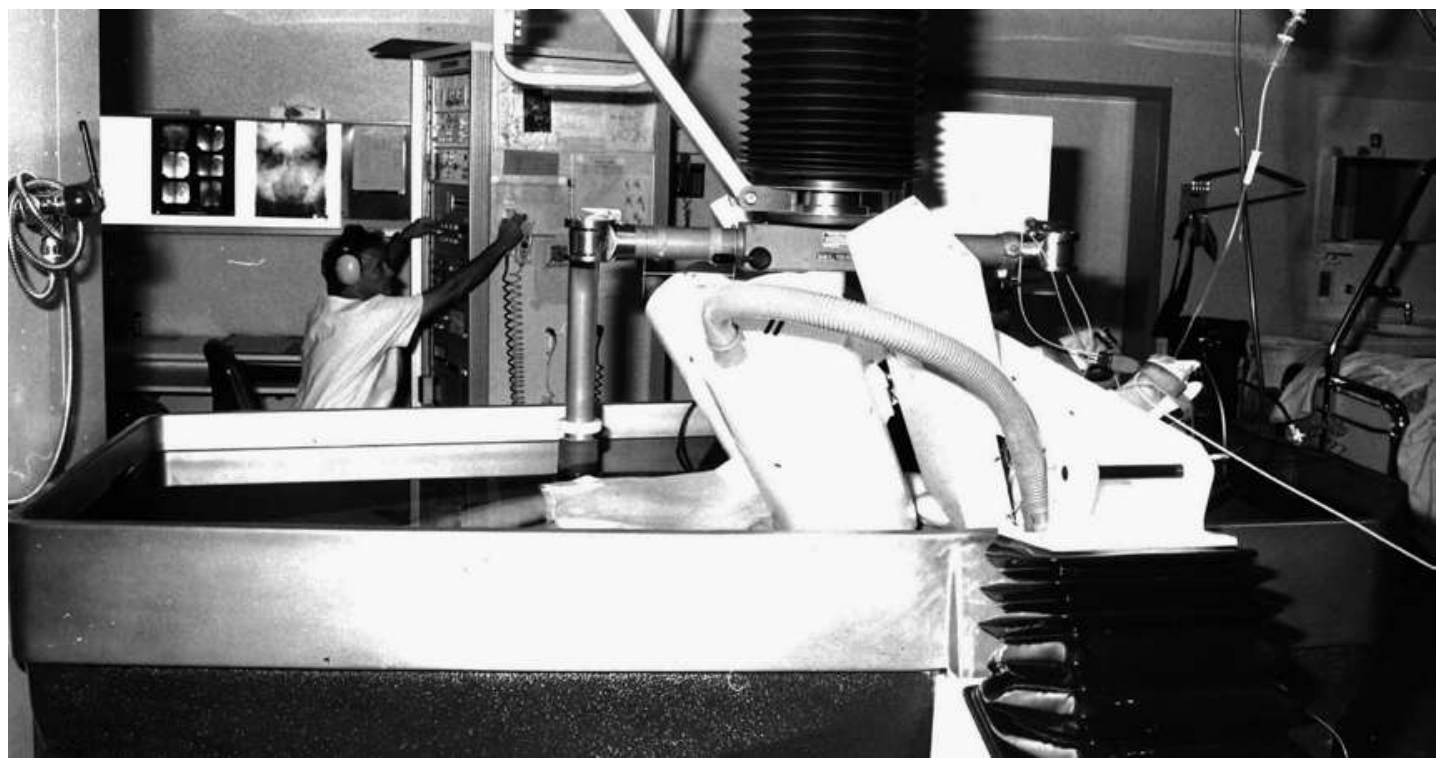

Der medizinisch-technische Assistent Rolf Schmutz während einer Behandlung mit dem Nierensteinzertrümmerer HM3, um 1990.

Mit Palmen, Liegestuhl, kühlem Bier und Grilladen wurde an einem schönen Sommertag des letzten Jahres ein eindrucksvolles medizinisches Gerät in den Ruhestand verabschiedet. Das Abschiedsfest für den Nierensteinzertrümmerer «HM3» (Human Model 3) - im Fachjargon «Extrakorporaler Stosswellenlithotripter» (ESWL) genannt - zog eine illustre Gästeschar an, die sich - wie fast dreissig Jahre zuvor zu seiner Einweihung - im «Steinkeller» der Universitätsklinik für Urologie des Inselspitals Bern und dem Garten davor versammelte, um die Maschine und ihre Leistungen zum Wohle einer Vielzahl von Patienten zu würdigen.

Bei insgesamt 17500 Patienten zertrümmerte das Gerät von 1985 bis Ende 2012 rund 48500 Nierenund Harnleitersteine. Und dies auf eine den Patienten schonende, nicht-invasive Art. Das Gerät prägte die Klinik über Jahrzehnte und trug erheblich zu deren Renommee und Wachstum bei. So erstaunt es nicht, dass den heutigen und ehemaligen Mitarbeitenden der Berner Urologie der Abschied vom Lithotripter schwerfiel.

Doch so endgültig war der Abschied nicht, denn der Nierensteinzertrümmerer fand nach dem Fest Eingang in die Museale Sammlung der InselspitalStiftung, wo er die Revolution in der Steinbehandlung für kommende Generationen dokumentiert.

\section{Vom Regentropfen zur Behandlungswanne}

Über Wellen zur Zertrümmerung von Nierensteinen wurde schon früh geforscht. Die Wirkung von Stosswellen und deren zerkleinernde Eigenschaften beim Auftreffen auf «feste» Materie wurde in der Luftfahrt, fernab der Medizin, entdeckt. Der entscheidende Durchbruch aber kam erst in den 1960er Jahren, als Ingenieure der Flugzeugfirma Dornier untersuchten, wie Regentropfen beim Überschallflug des Kampfjets «Starfighter» auf die Flugzeugstruktur einwirkten. Der Tropfenschlag erzeugte eine Stosswelle, die nicht nur an der Einschlagstelle, sondern auch im Innern des Materials Schäden verursachte. Treffen diese Stosswellen nun auf weiche Strukturen, werden sie weitergeleitet, ohne schwere Schäden anzurichten.

In einer Kooperation von Dornier mit dem Klinikum Grosshadern München gelang schliesslich die Innovation, die der Urologie den entscheidenden Fortschritt bringen sollte. Die Technik besteht im Kern aus einer grossen, wassergefüllten Badewanne, in die der Patient mittels Lift gehievt wird. Eine Durchleuchtungsanlage, die den Stein lokalisiert, hilft, den Patienten zu positionieren. Zwei Elektroden im Wasser am Boden der Behandlungswanne erzeugen die elektrohydraulischen Stosswellen mittels Funkenschlag. Die sich kugelförmig ausbreitenden Stosswellen werden mittels eines metallenen Ellip- 
soids ausgerichtet und auf den sich in Rückenlage befindenden Patienten fokussiert. Da Wasser dem menschlichen Gewebe sehr ähnlich ist, können die Stosswellen ohne grossen Wirkungsverlust oder Schädigungen an der Körperoberfläche in den Patienten eindringen und gelangen so zu Steinen im Bereich der Nieren und Harnleiter.

1980 wurde weltweit zum ersten Mal ein Patient erfolgreich mit dem Lithotripter behandelt. Zwei Jahre später galt die Behandlung offiziell als eingeführt. Mit der nicht-invasiven Behandlung von Nierensteinen wurde die bis anhin praktizierte offene Steinchirurgie auf einen Schlag überflüssig.

\section{In einer Kooperation von Dornier mit dem Klinikum Grosshadern in München gelang die Innovation.}

\section{Schnelle Berner}

Das Inselspital Bern war das erste Spital der Schweiz, das einen Nierensteinzertrümmerer kaufte. Die Projektabwicklung gilt als beispielhaft kurz und erfolgreich. Beim Lesen der Akten aus den 1980er Jahren spürt man noch heute die Euphorie für die damalige Anschaffung, gegen die es kaum Vorbehalte gab. Ernst Zingg war Chefarzt der Klinik für Urologie und Professor. Als Mitglied des Wissenschaftsrats bei Dornier verfügte er über einen Wissensvorsprung in Sachen ESWL. Dies brachte ihm auch einen Ruf an die Universitätsklinik Zürich, den er dann aber zugunsten Berns ablehnte, als sich hier die Anschaffung eines Nierensteinzertrümmerers abzeichnete. Im März 1983 orientierte Prof. Zingg den Verwaltungsrat des Inselspitals über diese neue Behandlungsmethode zur Beseitigung von Nierensteinen. Er präsentierte die Stosswellentherapie und belegte deren Wirtschaftlichkeit. Er begeisterte damit den anwesenden Regierungsrat derart, dass man gar von der Regel abwich, nie ein Gerät der ersten Generation anzuschaffen.

Von da an ging es Schlag auf Schlag. Schon im April 1983 wurde der Kauf im Grundsatz beschlossen. Argumente wie die «berührungsfreie Nierensteinzerkleinerung», die gesamtwirtschaftlich tieferen Kosten (kürzerer Spitalaufenthalt, weniger Dialyse) und die Deckung der Betriebskosten durch die Einnahmen überzeugten. Hochrechnungen sahen für das Inselspital jährlich ca. 440 Steinbehandlungen mittels Stosswellentherapie voraus. Ende 1983 beschloss der Insel-Verwaltungsrat definitiv, 5,4 Millionen Franken in den HM3 inklusive eines notwendigen Neubaus zu investieren. Im Februar 1984 bewilligte auch der Grosse Rat des Kantons Bern das Projekt mit 150:0 Stimmen. Der Insel-Bote, die Mitarbeiterzeitschrift des Spitals, verkündete daraufhin: «Wir sind sehr stolz auf dieses Projekt.» Am 1. März
1985, gerade einmal zwei Jahre nach der ersten Orientierung im Verwaltungsrat, konnte die Anlage eingeweiht werden.

\section{Pamir im Blitzgewitter}

Bereits einen Monat zuvor hatte sich der medizinischtechnische Assistent (MTA) Rolf «Röfe» Schmutz nach Berlin begeben, um dort die Bedienung des HM3 von Grund auf zu erlernen. Dieses Wissen und seine Erfahrung hat er stets weitergegeben. Seine HM3Demonstration anhand von Kunststeinen und seine legendären technischen Skizzen sind vielen in lebhafter Erinnerung geblieben.

Vor Beginn einer Steintherapie legte er jeweils zusammen mit dem zuständigen «Steindoktor» die Behandlungsstrategie fest und führte anschliessend die ESWL-Behandlung durch. 71 Urologen durchliefen in ihrem Curriculum während der Ära Schmutz den «Steinkeller» und nahmen mit Röfe Zertrümmerungen vor. Seine Erfahrung trug dazu bei, die ESWLTherapie zu verfeinern. So wurden in Bern Mitte der 1990er Jahre erstmals Kleinkinder behandelt, deren empfindliche Lungen mit Styropor und Alufolien vor streuenden Stosswellen geschützt wurden.

Für erwachsene Patienten verlief die Behandlung im Normalfall einfacher. Einige berichteten gar von einem ausgesprochenen Wohlbefinden während des «Beschusses». Im Patientenlift würde man wie in einem Schaukelstuhl liegen, man werde via Kopfhörer mit Musik berieselt, und das wohlig warme Wasser in der Wanne vermittle dank Auftrieb ein leichtes Gefühl, zu dem wohl auch die verabreichten Schmerzmedikamente beitrugen.

Für den behandelnden Urologen und den MTA Röfe Schmutz bedeutete jede Behandlung sorgfältige Arbeit und volle Konzentration. Mit dem Pamir-Gehörschutz auf den Ohren, um den klopfenden Lärm des HM3 abzumildern, und eingetaucht in das gespenstische Licht der blitzenden Elektroden, überwachten sie laufend die Parameter und nahmen gewissenhaft Einstellungskorrekturen am Bedienturm vor.

\section{Mit Hybrid zum Erfolg}

Schon bald nach Einführung des HM3 wurde die Urologische Klinik von der Nachfrage überrannt. In den ersten zehn Monaten waren bereits 606 Patienten damit behandelt worden. Zur Bewältigung der fortan knapp 600 Steinpatienten pro Jahr wurden weitere Lithotripter angeschafft, um alternierende Behandlungen mit folglich kürzeren Wechselzeiten zu ermöglichen. Keines der moderneren, elektromagnetisch oder piezoelektrisch arbeitenden Nachfolgermodelle vermochte aber mit der klinischen Effizienz des HM3 Schritt zu halten, wie diverse Publikationen der Berner Universitätsklinik für Urologie belegen. Darunter ist auch eine der wenigen prospektiv randomisierten Lithotripter-Vergleichsstudien. Sie wurde mit 820 rekrutierten Patienten durchgeführt [1]. 


\section{Übersichtsschema - Diverse Varianten}

Wo beginnt man mit der Steinzertrümmerung?

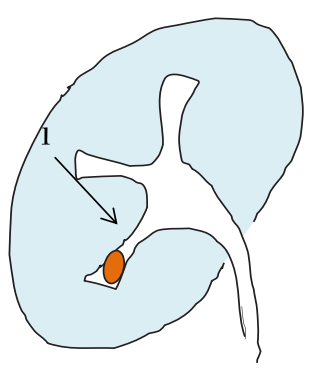

Unterer

Kelchstein

Beginn siehe

Pfeil 1

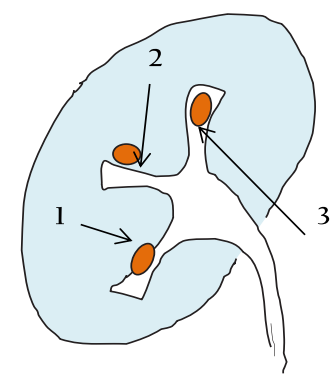

Unterer / Mittlerer-

und Oberer Kelchstein

\section{Beginn siehe}

Pfeil $1 / 2 / 3$

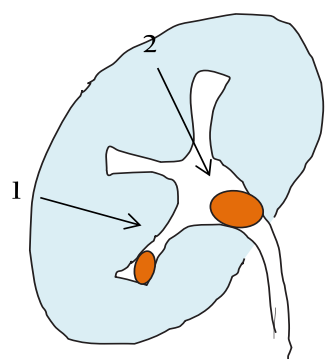

Peylon und

Unterer

Kelchstein

Beginn siehe

Pfeile 1/2
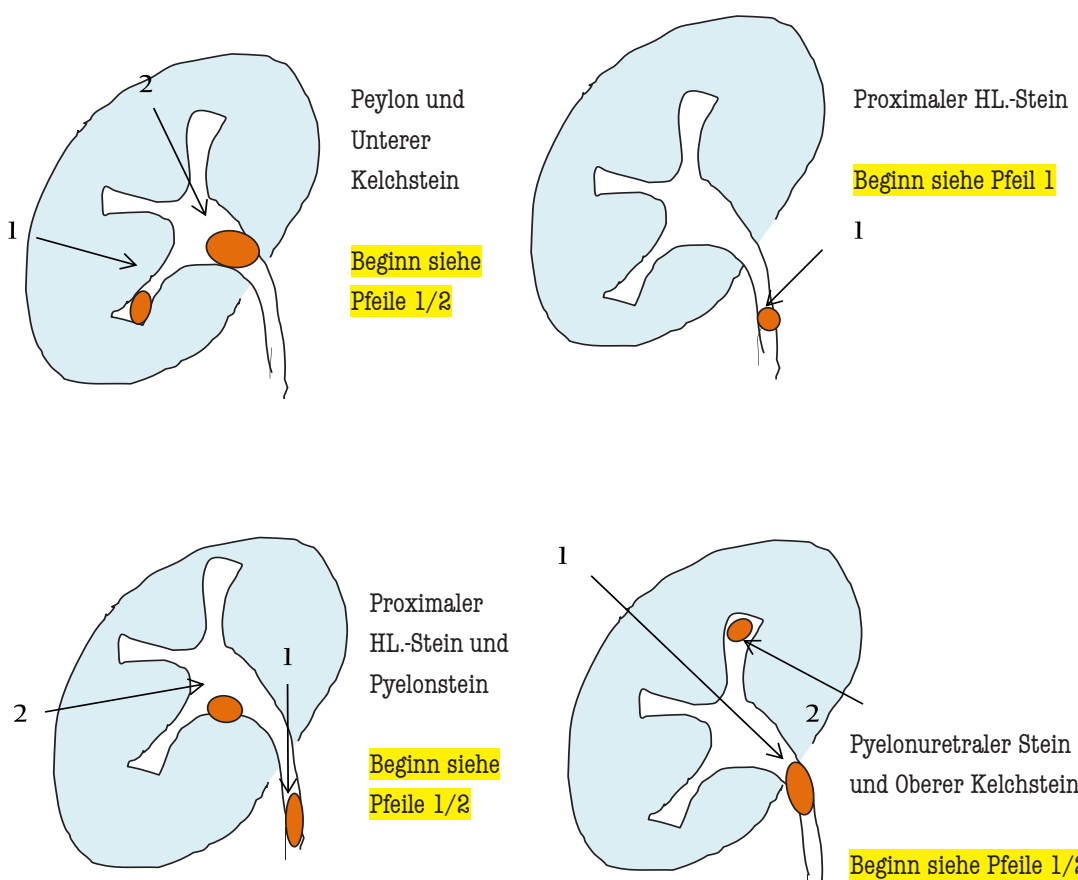

Proximaler

HL.-Stein und

Pyelonstein

Beginn siehe

Pfeile 1/2

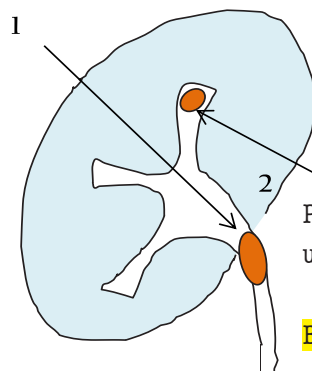

Pyelonuretraler Stein

und Oberer Kelchstein

Beginn siehe Pfeile 1/2

Anleitungsskizze des MTA Rolf Schmutz für unterschiedliche Vorgehensweisen bei der Steinzertrümmerung.

1 Zehnder P et al. A Prospective Randomised Trial Comparing the Modified HM3 with the MODULITH SLX-F2 Lithotripter. In: European Urology. 2011;59(4):637-44
Der beachtliche Behandlungserfolg des Berner Lithotripter HM3 beruhte auf seinen technischen Eigenschaften. Er war nämlich eines der seltenen sogenannten «Hybrid-Modelle». In den 1990er Jahren wurde der originale HM3 modifiziert, um Behandlungen auch ohne Narkose zu ermöglichen. Dafür wurde bei den Geräten nachträglich einerseits die ellipsoide Öffnung von $176 \mathrm{~cm}^{2}$ auf $232 \mathrm{~cm}^{2}$ vergrössert, um die Stosswellen-Energie auf eine grössere Hautoberfläche zu verteilen. Gleichzeitig halbierte man aber auch die Kapazität des Stosswellen-Generators auf $40 \mathrm{nF}$ (Nanofarad). Die technischen Veränderungen hatten eine Abnahme der StosswellenEnergie im Behandlungsfeld und somit Verminderung der Schmerzen zur Folge. In Bern vergrösserte man aber nur das Ellipsoid, den Stosswellen-Generator beliess man bei $80 \mathrm{nF}$. Es resultierte der sogenannte «Hybrid-HM3». Bedenkt man die Atemverschieblichkeit der Nieren von mehreren Zentimetern, so wird klar, dass der grössere Stosswellenfokus des Hybrid-HM3 (ca. 5 Franken-Stück) mit zudem hoher Schlagkraft u. a. aufgrund der besseren Trefferwahrscheinlichkeit zu einer effektiveren Zertrümmerung des Steines führt als die zunehmend präzisere Fokussierung der Neuentwicklungen.

Ungeachtet der eindrücklichen klinischen Effizienz mussten am 17.12.2012 die Behandlungen mit dem letzten HM3 der Schweiz (und sogar einem der letzten weltweit) eingestellt werden. Der zunehmende Mangel an Ersatzteilen und das Alter des Materials gaben den Ausschlag, ganz im Sinne der Patientensicherheit. Die HM3-Ersatzbeschaffung ist in vollem Gange. Die elektrokonduktive Stosswellen-Erzeugung des neuesten Lithotripters ist mit der elektrohydraulischen des HM3 verwandt. Diesen sechsten Lithotripter an der Urologischen Universitätsklinik wird der MTA Rolf Schmutz nur noch bis Ende 2014 bedienen. Nach 29 Jahren «Steinkeller» mit Behandlung von über 23000 Steinpatienten wird er den wohlverdienten Ruhestand antreten. Nicht aber bevor er sein immenses Know-how über Apparate und Behandlungsstrategien an seine Nachfolger weitervermittelt hat.

\section{ESWL reloaded}

Doch damit ist die Geschichte noch nicht zu Ende: Vier Monate vor der geplanten Ausserbetriebsetzung der geschichtsträchtigen Maschine erfuhr David Sharp, ein freiwilliger Mitarbeiter der Musealen Sammlung des Inselspitals davon und informierte die Sammlungsleitung darüber. Er selbst hatte 1985 für kurze Zeit mit dem Gerät gearbeitet und empfahl, es in die Sammlung aufzunehmen. Auch das Science Museum in London zeigte Interesse am Gerät. Doch Bern erhielt den Zuschlag.

Wie bereits 27 Jahre zuvor breitete sich das ESWLVirus von Neuem rasant aus. Es folgten Abklärungen und Besichtigungen der Maschine, Recherchen zur historischen Bedeutung, Verhandlungen und Analysen zum Transport. Die Museale Sammlung schaffte Platz für die riesige Badewanne, Vertreter der Universitätsklinik für Urologie sammelten Dokumentationsmaterial und stellten sich für Interviews zur Verfügung. Der Abund Wiederaufbau wurde von der Insel-Medizintechnik in Zusammenarbeit mit dem damaligen Hersteller Dornier MedTech GmbH minutiös geplant. Am 11. Juli 2013 schliesslich stand der Nierensteinzertrümmerer HM3 an seinem neuen Ort in den Sammlungs-Räumlichkeiten und wurde im Oktober in Anwesenheit der einstigen und heutigen Protagonisten eingeweiht. Die altgediente Maschine hatte uns alle noch einmal zu Koordinations-Höchstleistungen angespornt.

Heute ist der Nierenlithotripter nicht nur das eindrücklichste Sammlungsobjekt in der Musealen Sammlung der Inselspital-Stiftung, sondern auch das bestdokumentierte. Hier wird es konserviert und interessierten Menschen heute und morgen zugänglich gemacht. Es bezeugt beispielhaft den Paradigmenwechsel in einer Therapiemethode der Medizin und steht für eine faszinierende Entwicklung in der neueren Geschichte des Inselspitals Bern. 